\title{
Drone-based Cooling Leakage Measurement from Glass Front Towers
}

Hadeel Abed, Nada Abdou and Nizar Zorba

Department of Electrical Engineering, Qatar University

\begin{abstract}

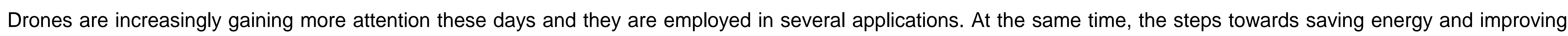

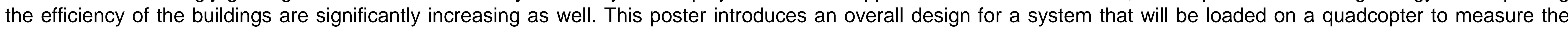

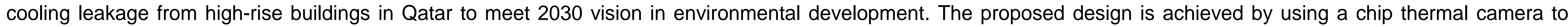

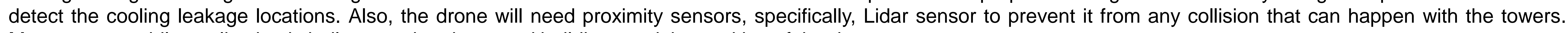
Moreover, a mobile application is built to monitor the tested buildings and the position of the drone.
\end{abstract}

\section{Introduction}

Drones came into first use in 1916 for military purposes in The US during the World War I [1]. Nowadays, drones have different use cases, especially those related to environment and agriculture. For instance, a previous study in Qatar used a drone to monitor and sense air quality and pollution parameters [2]. Another application was using a drone for livestock monitoring and tracking in fields [3]. The focus on this work is to use the drones for environment and energy efficiency. Qatar has been ranked between the highest per capita in energy supplying (236 MWh/capita), and in emitting greenhouse gases, such as, $\mathrm{CO} 2$ (35.7 tons) [4]. Krarti et al. [5] stated that in Qatar, the buildings sector including residential, commercial, and governmental buildings occupy $58 \%$ of the total electricity consumption in 2014. In 2018, Air conditioners are consuming from $60 \%$ to $80 \%$ of the electricity in Qatar. Apparently, there are air conditioners in every single building in Qatar, and they are operating 24/7 during summer [6]. To achieve Qatar vision 2030, the Qatari government launches plenty of programs and awareness campaigns for electricity and water saving, such as Tarsheed by Kahrmaa in 2012 that aim to minimize the electricity consumption to 39 $\mathrm{KWh} /$ capita. Moreover, the gulf organization for research and development (GORD) and university of Pennsylvania have come up with a system for rating the sustainable building. This rating system has been called Qatar Sustainable Assessment System (QSAS) at the very beginning, but nowadays, it is Global Sustainable Assessment System (GSAS). The system helps the designers to ensure that their buildings are eco-friendly as much as possible, in terms of materials, energy, water, indoor environment, etc. [5].

\section{System Design}

To begin with, to design the system that will be used to find the locations of cooling leakage at different levels of towers by the drone, one need to select the most suitable components. The process of selecting the components was to compare different types of microcontrollers, chip thermal cameras, and different proximity sensors, such as ultrasonic and Lidar based on different criteria, such as accuracy, cost, weight, and response time, etc. After the comparison has been done, the selected components were Raspberry Pi 3 model B, Flir Lepton 2.5 thermal camera, and four Lidar Lite V3 sensors. Raspberry $\mathrm{Pi} 3$ is used for collecting the data, and send it to the ground station through $4 G$ network. The drone will be piloted in an autonomous way, and this could be done by using GPS and open source software, such as mission planner. The overall design is shown in Figure 1. The sensors have been tested as well as the main component that is the thermal camera. Figure 2 shows its results.

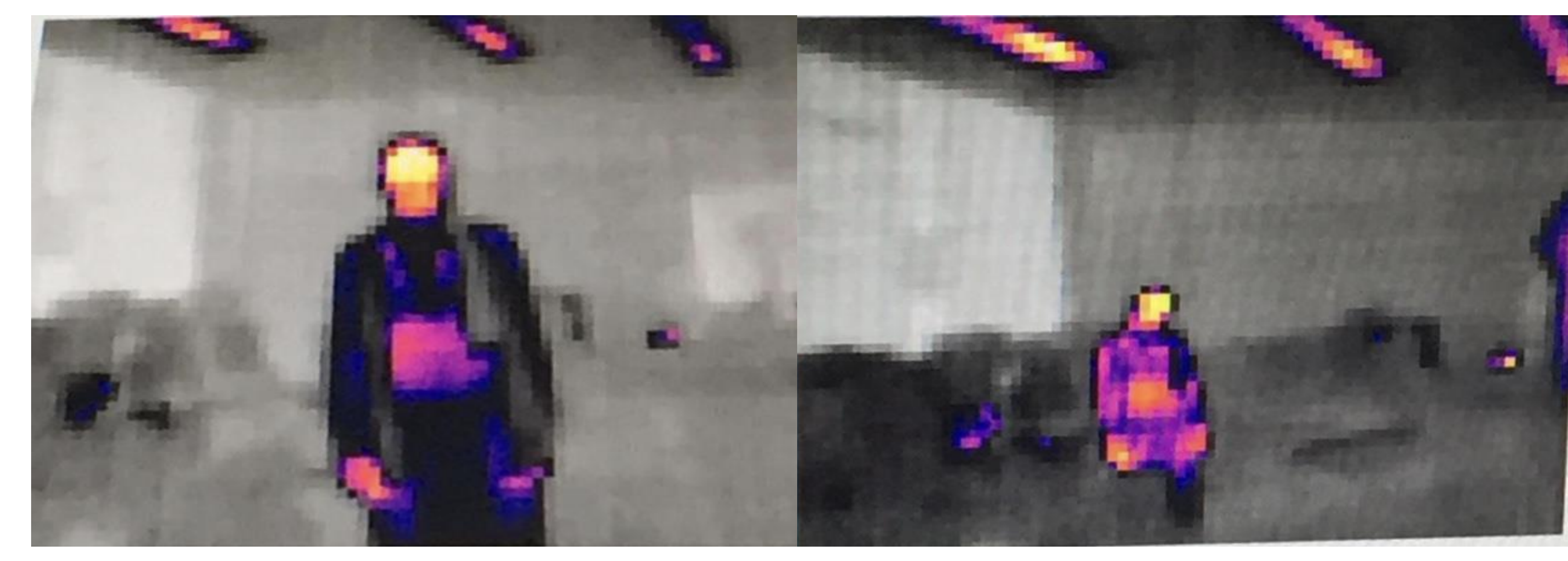

Figure 2. Lepton 2.5 Testing

\section{Conclusions and Future Work}

A system is designed for a drone to fly and measure the cooling leakage from high-rise building in Qatar using a Flir chip thermal camera. In addition, the drone will be provided with Lidar sensors to avoid collisions. The brain of the system is the Raspberry Pi 3 to process the images from the thermal camera. The overall block diagram has been shown. For the future work, a permission is needed to be received to fly the drone near to the towers. Also, increasing the capabilities and features of the mobile application. Finally, the results will be reported to interested parties such as Kahramaa.

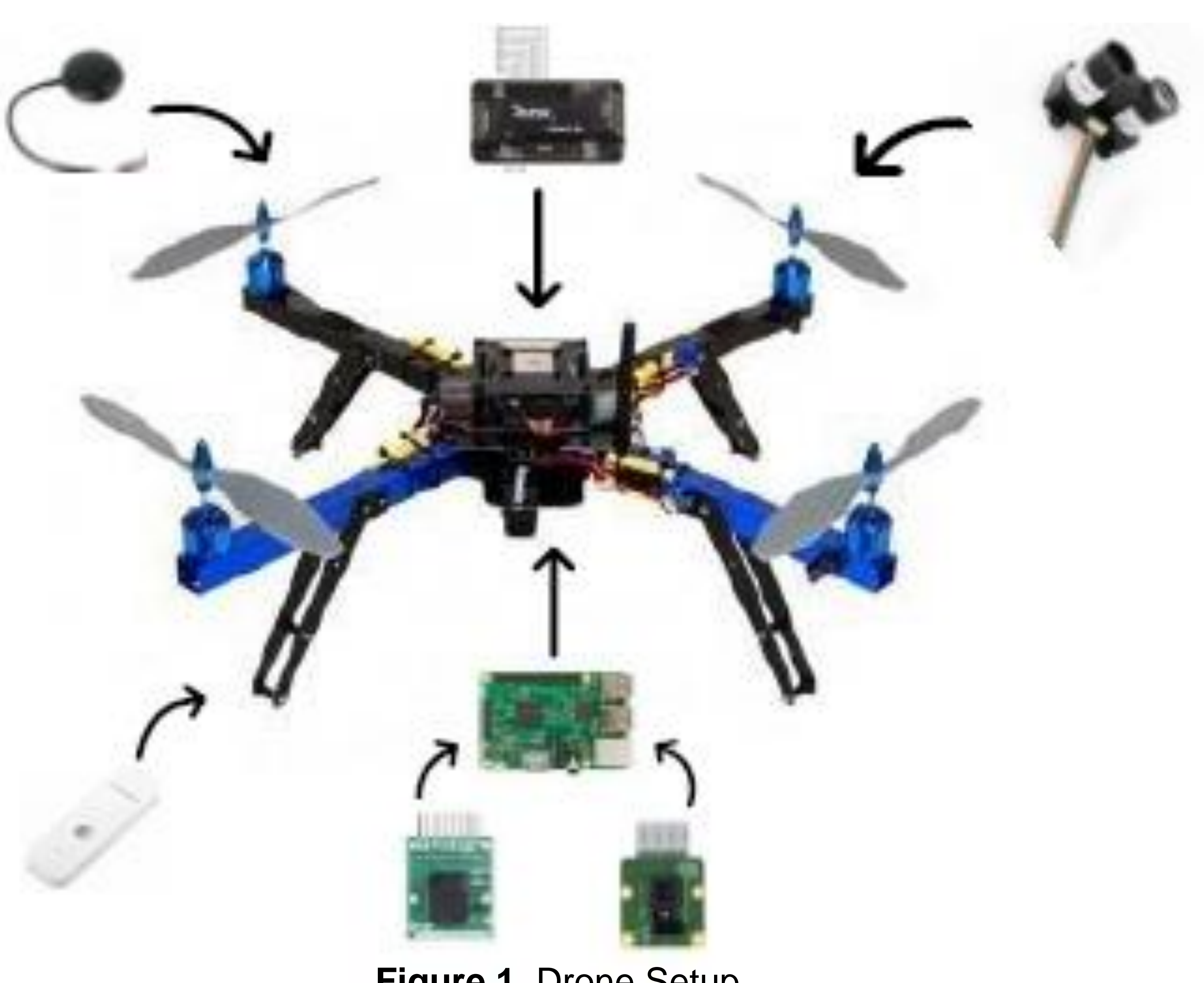

Figure 1. Drone Setup.

\section{Mobile Application Results}

In order to better show the project outcomes, an Android mobile application has been developed to monitor the sensors readings and for camera live streaming. The application allows its users to choose between different buildings and adding new will be connected to Firebase database to store and read data from buildings for testing, then, it will provide the user with real-time readings from the system sensors and modules. The application has been built using Android Studio. The application takes the sensors readings in real-time bases. For drone safety, its position can be monitored using the App, and the app will notify the user when the drone is close to the tower. Figure 3 illustrates an example when the drone is close and when it is far away from the building.
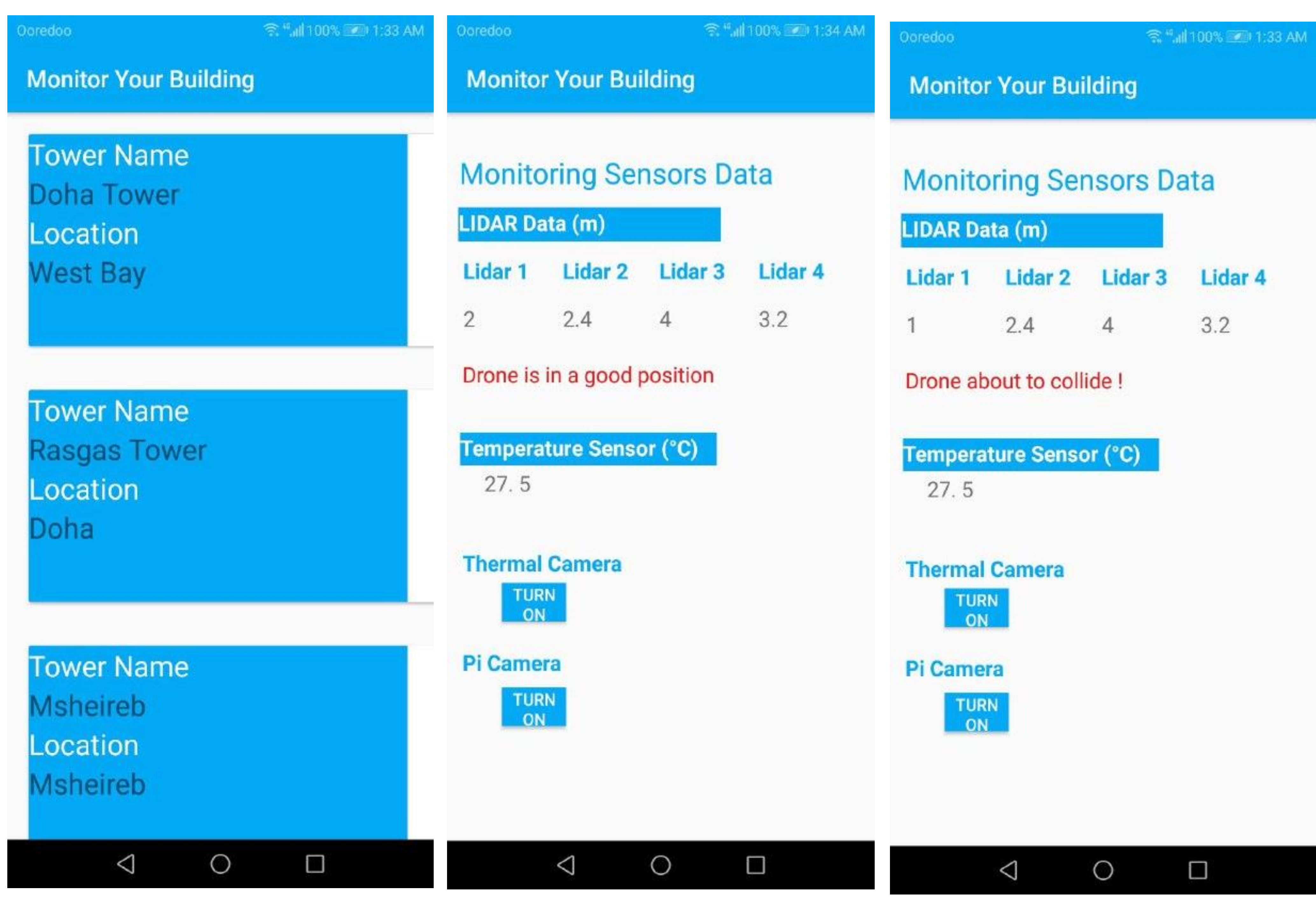

Drone is in a good position
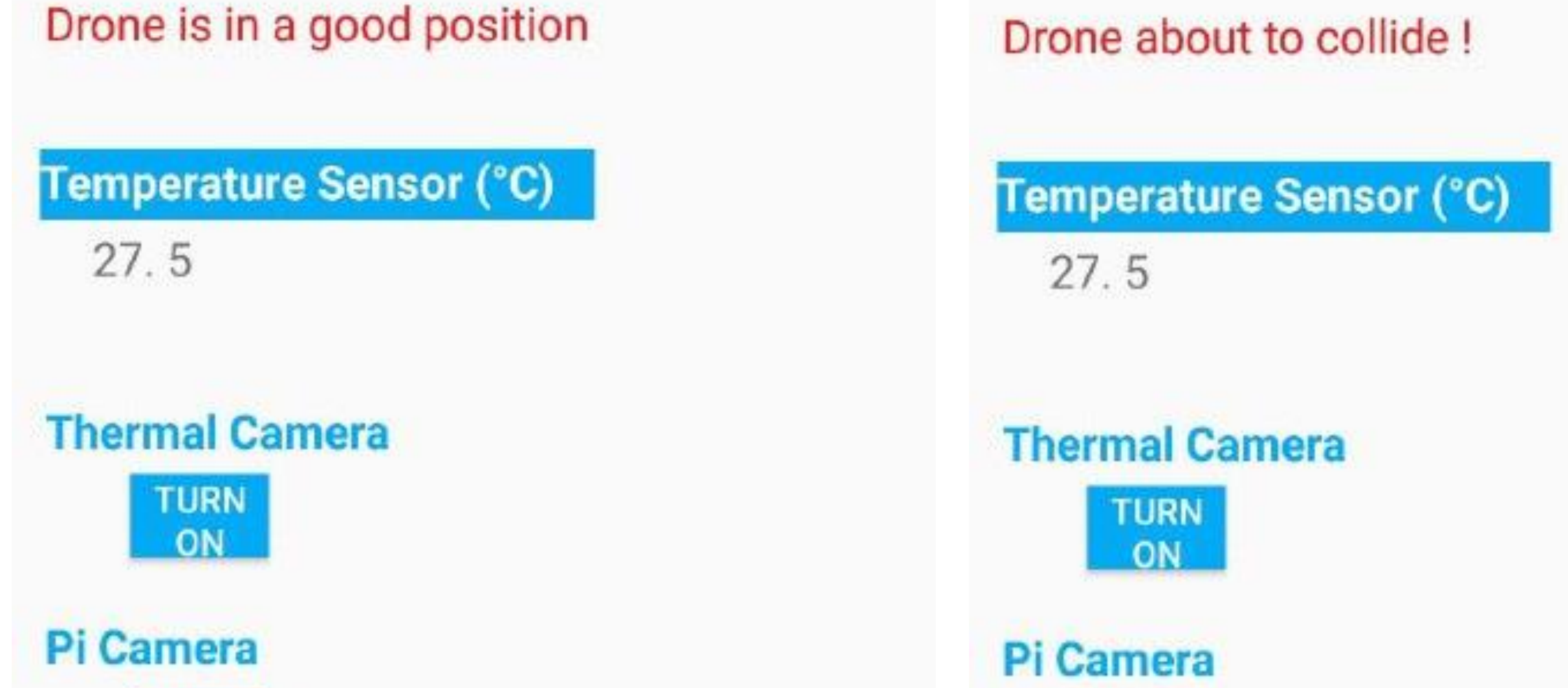

TURN

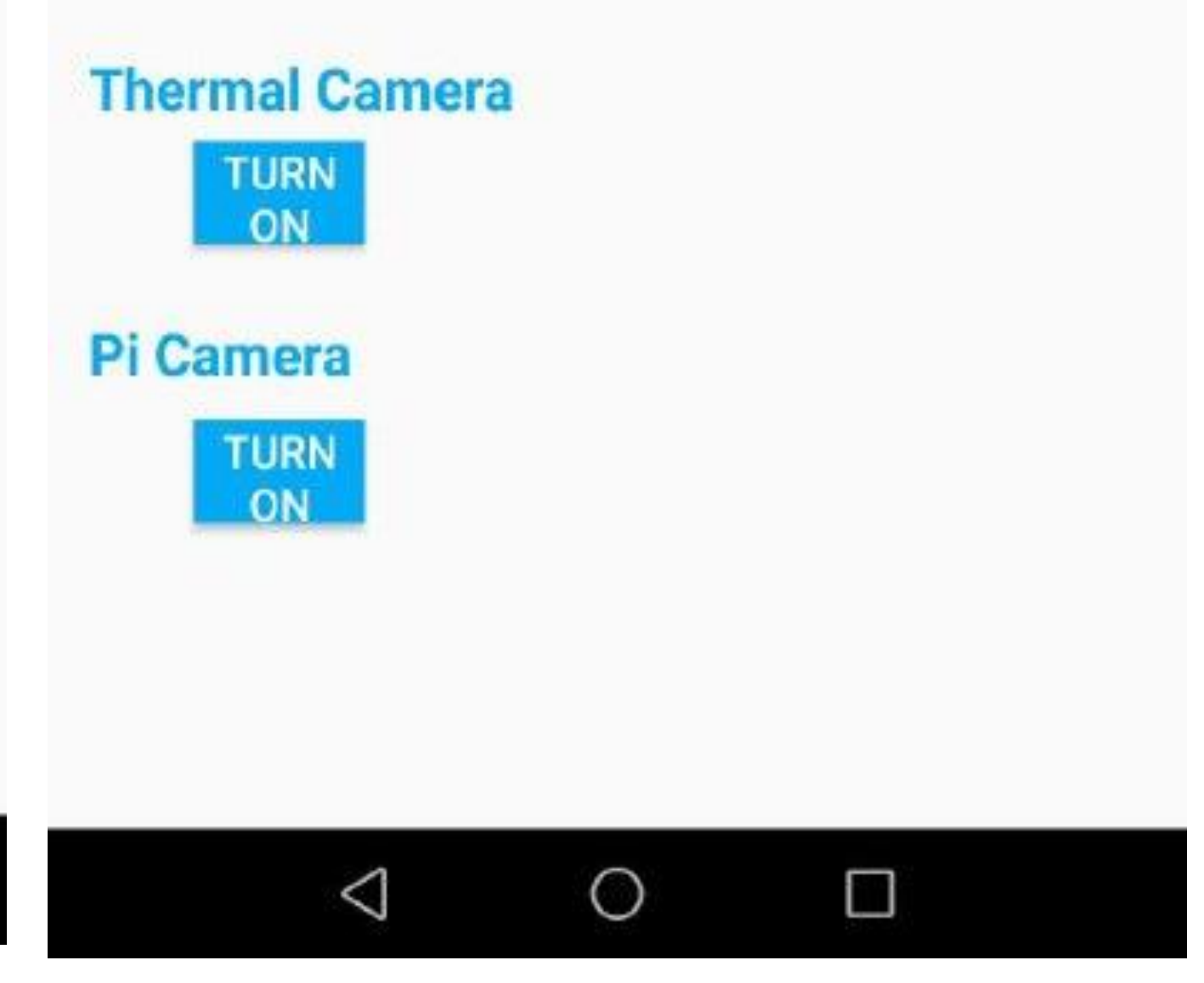

Figure 3. Mobile Application

\section{Achievements}

This project with the supervision of Prof. Nizar Zorba won the 3rd place at the 7th Innovation and Entrepreneurship Contest at Qatar University.

\section{References}

